\title{
Cosmic web imager
}

Shahinur Rahman, Chris Martin, Ryan McLean, Matt Matuszewski, Daphne Chang

Shahinur Rahman, Chris Martin, Ryan McLean, Matt Matuszewski, Daphne Chang, "Cosmic web imager," Proc. SPIE 6269, Ground-based and Airborne Instrumentation for Astronomy, 62693W (29 June 2006); doi: $10.1117 / 12.672732$

SPIE Event: SPIE Astronomical Telescopes + Instrumentation, 2006, Orlando, Florida , United States 


\title{
Cosmic Web Imager
}

\author{
Shahinur Rahman*, Chris Martin, Ryan McLean, Matt Matuszewski, Daphne Chang \\ California Institute of Technology, 1200 E. California Blvd., Pasadena, CA, USA 91125
}

\begin{abstract}
We are developing the Cosmic Web Imager (CWI) to detect and map emission from the intergalactic medium (IGM). CWI will observe the strong, redshift UV resonance lines of Lya 1216, CIV 1550, and OVI 1033 over 3600-9000 $\AA$ to trace IGM at $1<\mathrm{z}<7$. CWI is an integral-field spectrograph designed for the Hale Telescope at Palomar Observatory. CWI combines in a novel way three mature and extensively used instrumental techniques. The Integral Field Unit (IFU) provides a wide 2D field of view of $60 \times 40$ arcsec 2 for observing extended emission over a large region. The spectrograph using Volume-Phase Holographic gratings have high peak diffraction efficiency and are tunable for covering a large bandpass with a single grating. A low read noise CCD combined with source/background shiftand-nod allowing control of systematics and Poisson-limited sky subtraction to observe the low surface brightness universe. With a resolution of $\mathrm{R}=10,000 \mathrm{CWI}$ is sensitive to limiting surface brightness ranging from $25-27.5 \mathrm{mag} / \operatorname{arcsec} 2(10 \mathrm{~min}-8$ hours integration). Recent high resolution simulations predict Ly $\alpha$ Fluorescence from IGM at $100-1000$ LU1. CWI with sensitivity of $\sim 200 \mathrm{LU}$ improves the current observational effort by an order of magnitude and enables us to explore wide range of overdensity $(\delta \sim 30-104)$ testing the standard model of structure formation in the universe. CWI also serves as the counter part to the balloon borne integral-field spectrograph Faint Intergalactic medium Redshifted Emission Balloon (FIREBALL) currently being built and planned to be launched in Summer 2007. FIREBALL will observe Ly $\alpha$ Fluorescence from IGM at $\mathrm{z}=0.7$. CWI combined with FIREBALL will enable us to observe the evolution of IGM and the low surface brightness universe.
\end{abstract}

Keywords: IGM, Palomar

\section{SCIENTIFIC OBJECTIVES}

Ever since Ly $\alpha$ forest, which is the absorption feature lines corresponding to the Ly $\alpha$ rest wavelength, has identified as clouds of IGM intersecting along the line of sight it has been used to study IGM. In 1990s spectra of quasar pairs showed coherence over scales of a hundred kpc and more, implying large sizes and low densities for the absorbing structure in accordance with $\Lambda \mathrm{CDM}$ cosmological simulations. Qualitative questions concerning the nature and interpretation of IGM has given way increasingly to quantitative investigations of astrophysical properties of the baryon field, such as temperature, metallicity, kinematics, radiation field and dependence on the underlying cosmological parameters. However as Figure 2 shows, information on individual line of sight obtained from single line of sight does not reveal the complex astrophysical properties of IGM.

It is only possible to reveal the 3D structure of IGM through observing Lya Fluorescence and other metal line emissions. With the 3D knowledge of IGM we can study following list of astronomical questions.

- Galaxies form from the overdense regions of IGM, therefore, IGM emission mapping will reveal the nature of galaxy formation and its interaction with IGM.

- Simulations have shown that diffuse is an excellent tracer of large-scale distribution of dark matter. A large effort is underway to measure cosmological structure by QSO absorption line statistics. However, these measurements are fundamentally limited by the large cosmic variance resulting from isolated QSO line of sight. IGM emission mapping will vastly reduce this variance and will provide a detailed look at the primordial power spectrum and physics of inflation.

*shahin@caltech.edu; phone 1 626 395-4787; fax 1626 568-0285

Ground-based and Airborne Instrumentation for Astronomy, edited by lan S. McLean, Masanori lye, Proc. of SPIE Vol. 6269, 62693W, (2006) · 0277-786X/06/\$15 · doi: 10.1117/12.672732 
- Correlation of IGM absorption features with emission features will enable the comparing of fiducial lengths in velocity (redshift) and linear (angle) space to measure deviations of the Hubble expansion from EdS (AlcockPaczynski Test).

- The majority of baryons do not reside in galaxies but are believed to reside in IGM. Locating of IGM and therefore baryons will provide an independent measurement of the baryon density and will confirm or refine the standard model of structure formation.

- Measurement of metal abundances will give important clues feedback of energy and metals by star forming galaxies, which are key uncertainty in cosmological simulations.

The detection and exploitation of CMB fluctuations, perhaps the most import cosmological measurement done to date, required dedicated instruments and long term efforts to improve technique. IGM mapping is at least a decade behind other cosmological tools to study the structure of the universe. CWI will be first of its kind dedicated to mapping the IGM. Another major application of CWI will be to measure the low surface brightness universe. For example, galaxies like NGC5907 we propose to measure as far as 40-50 kpc above the disk. This will map the dark halo profile almost two order of magnitude in radius. No other extent or planned instrument combines high spectral resolution, wide field of view, and sensitivity to extremely low surface brightness emission as CWI.

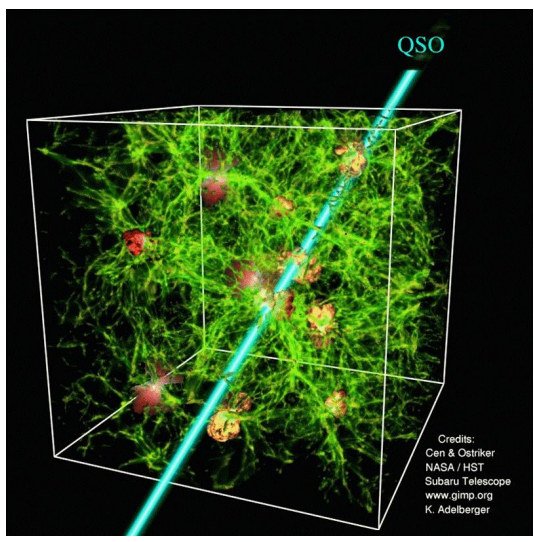

Fig. 1. Simulation showing 3D structure of IGM with a line of sight to a QSO. The red regions are IGM polluted by metalrich super winds showing complex structure not observable with single line of sight. ${ }^{1}$

\section{INSTRUMENT AND OBSERVATION}

In order to achieve the scientific goals, the instrument design requires that we have high resolution, wide 2D field of view, simultaneous band and field of view coverage, and precision sky subtraction and control of systematics. High resolution is required to provide the best possible source/background subtraction of complex nightglow and zodiacal light spectra, sufficient contrast between faint IGM lines and the continuum sky background, to resolve IGM lines from complex continuum and airglow band features, and to kinematically separate IGM/PGM components. A wide, $\sim 2400$ $\operatorname{arcsec}^{2}$ field of view is required to provide excellent sensitivity to extended, low surface brightness emission. As we show in Figure 2, bright IGM emission regions are predicted to have typical angular extents of several tens of arcseconds (several hundred kiloparsecs). A long slit spectrograph would need to be scanned to survey a region of this dimension, which precludes obtaining the deepest possible exposure on a single region. Spectrographs extant on large telescopes are optimized for sub arcsecond PSFs and have modest fields of view when operated with IFUs. The CWI long-slit spectrograph is fed by an Integral Field Unit (IFU) to provide a $60 \times 40 \operatorname{arcsec}^{2} 2 \mathrm{D}$ field of view sampled by $24 \times 2.5$ arcsec reflective slits. This format is illustrated in Figure 2. This format is also ideal for probing galactic winds in distant galaxies and for kinematic mapping of stellar halos. In our application, a broader band spectrum is desirable since metal line complexes may have multiple components spread over $500 \mathrm{~km} / \mathrm{s}$ or more, metal lines are usually doublets (CIV, OVI) and faint lines will be confirmed only by detection of both features, which are separated by several angstroms. A moderate band of the spectrum provides important information about the various background sources and whether they are being effectively subtracted. With the integral field spectrograph design we can obtain a very deep exposure over the bandpass without scanning, and also perform a survey type observation. CWI will be designed to minimize systematic 
errors in background subtraction. Airglow background in particular varies over the sky, in wavelength, and in time during the observing night. The airglow in the $3500-4500 \AA$ band that we baseline for initial observations is dominated by $\mathrm{O}_{2}$ (Herzberg and Chamberlain bands) and weak $\mathrm{OH}^{2}$. These all exhibit spatio-temporal variations due to gravity waves and turbulence propagating through the layer in which the photochemical reactions produce the observed bands ${ }^{3}$. The observational strategy must be optimized to either measure or average over these variations. Instrumental nonuniformities when combined with variable backgrounds complicate this objective. That the source and background are both extended over large, common angular regions is a further annoyance. The scanning/chopping strategy is critical, as is the control of systematic errors.

With the above systems requirements, we have developed an instrument design with an IFU, collimator, VPH grating, Norris camera, and $4 \mathrm{k} \times 4 \mathrm{k}$ Lesser CCD as shown in the raytrace of Figure 3.
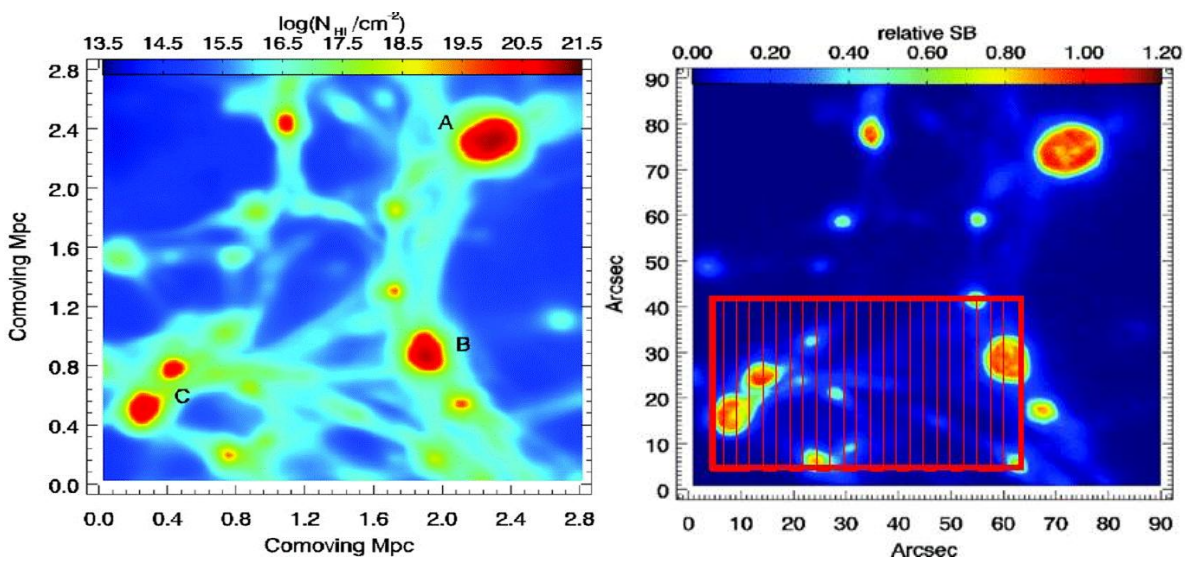

Fig. 2. Left Panel is Column-density distribution of neutral hydrogen at $z=3$, the gas is exposed to a diffuse UV background generated by the population of galaxies and quasars. Right Panel is broad band images ( $\sim 90 \AA$, centered on $4864 \AA$ ) of fluorescent Ly $\alpha$ emission at $\mathrm{z}=3$ for static gas clouds relative to $\mathrm{SB}=3.67 \times 10^{-20} \mathrm{erg} \mathrm{cm}^{-2} \mathrm{~s}^{-1} \operatorname{arcsec}^{-2} \sim$ $360 \mathrm{LU}$. Overlaid in red on the right panel is the field of view and slits of the IFU. CWI will have sensitivity limit extending to yellow-green region. ${ }^{4}$

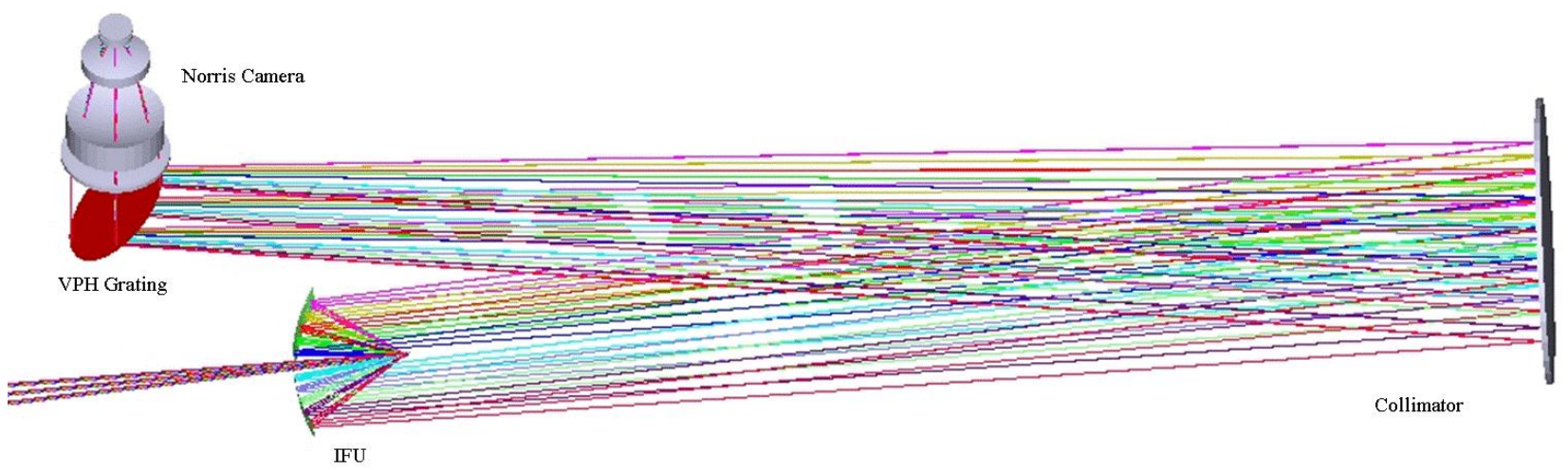

Fig. 3 Raytrace excluding the telescope.

\subsection{IFU}

The long-slit spectrograph is fed by an Integral Field Unit (IFU)to provide a $60 \times 40 \operatorname{arcsec}^{2}$ field of view sampled by 24 x 2.5 arcsec reflective slits. 24 reflective planar mirrors, each $1 \mathrm{~mm}$ by $15 \mathrm{~mm}$, will redirect the diverging f/16 Cass beam to 24 planar mirrors positioned on a hyperboloidallocus, with total height of $38 \mathrm{~cm}$, in order to preserve the optical path and pupil. The baseline design incorporates a set of diamond-turned post-polished Aluminum flats assembled and 
aligned to give the correct angular redirection. Figure 4 shows 10 incoming rays corresponding to fields along y direction, transformed into a long slit in $\mathrm{x}$ direction. The red disc on the left correspond to the location of virtual slit.

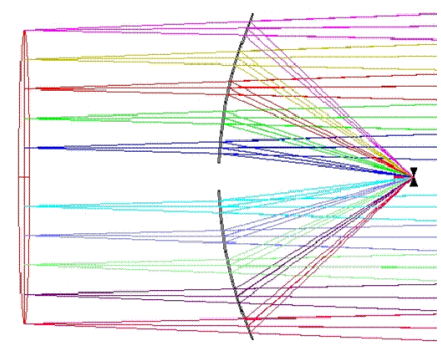

Fig. 4 Raytrace of IFU.

\subsection{VPH Grating Spectrograph}

The spectrograph design is driven by the requirement of long slit, $\mathrm{R}>5000$, high efficiency, and achromatism over a $10 \mathrm{~nm}$ band with a band center range of 350-900 $\mathrm{nm}$. The recent development of Volume Phase Holographic (VPH) gratings ${ }^{5}$ is an alternative to the conventional surface ruled grating. VPH gratings diffract light by fringes or refractive index variations within the volume rather than the periodic surface structure in surface ruled gratings. As seen in figure 5 , which is calculated efficiency of $6 \mathrm{VPH}$ gratings, they have a high peak efficiency over a wide bandpass. Also the transmissive nature of VPH grating has allowed us to design a camera with minimal vignette.

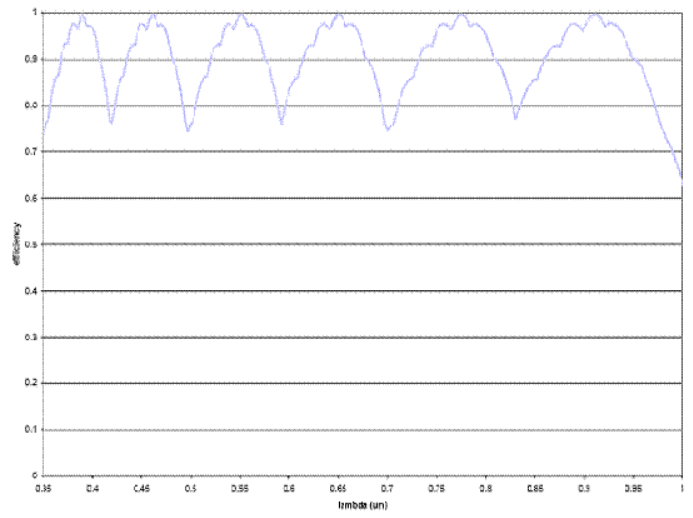

Fig. 5 Efficiency vs. wavelength for 6 VPH grating. Covering our band pass of $350-900 \mathrm{~nm}$.

\subsection{Camera Design and CCD}

Our requirement for the camera is to have a large entrance pupil with high throughput over the bandpass of $350-900 \mathrm{~nm}$ having spot size less than the slit width of 100 micron. A camera satisfying these conditions have been built for the Norris Spectrograph for the Hale Telescope at Palomar Observatory. The Norris camera was designed by Epps ${ }^{6}$ and the optical prescription can be found in Figure 6. This camera accepts 6 inch entrance pupil and accommodates the full wavelength interval of $350-900 \mathrm{~nm}$ with a spot size less than 80 micron allowing refocusing by shifting the CCD. Some spot sizes are shown in Figure 7. The use of Norris Camera significantly reduces the time and cost of building the instrument. However, the throughput and optical performance of the camera is yet to be tested.

For CCD we plan to use Lesser 60 x $60 \mathrm{~mm}$ UV enhanced 4096 x 4096 pixel CCD with $15 \mathrm{~mm}$ pitch and $2.5 \mathrm{e}^{-}$read noise. The Palomar staff has had a favorable experience with the device and report a QE of $75 \%$ at $350 \mathrm{~nm}$. The low read noise $2.5 \mathrm{e}^{-}$is critical for making the high resolution spectra sky noise limited in a reasonable amount of time. In survey mode (see below) this occurs in about 10 minutes using 4 by 4 pixel binning. In deep mode ( 2 by 2 binning) this occurs after about one hour. 


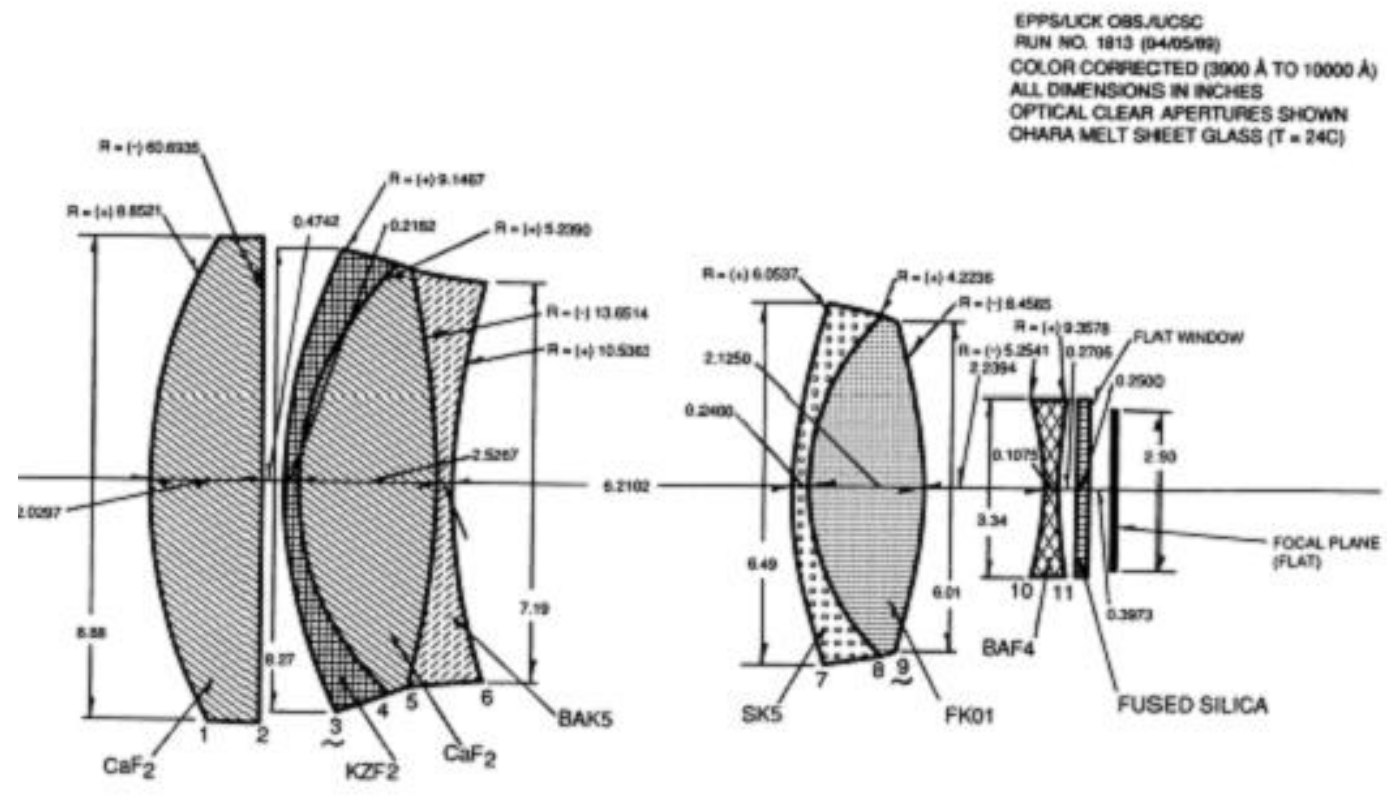

Fig. 6. Norris Camera
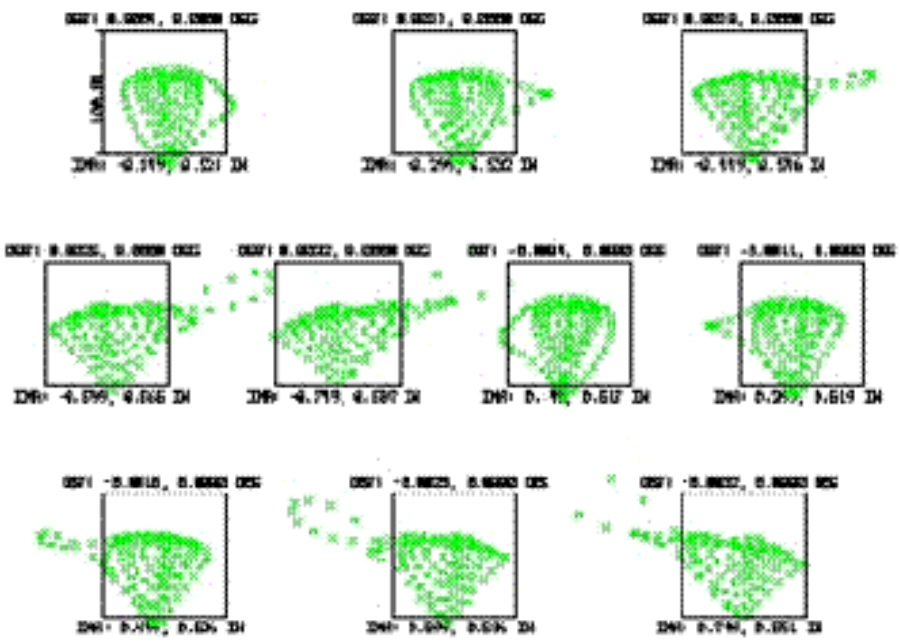

Fig. 7. Spot Diagram at $400 \mathrm{~nm}$ with $100 \mathrm{~mm}$ box

\subsection{Source/Background Shift and Nod}

We will adopt the shift and nod strategy ${ }^{7}$ as shown in Figure 8. In this scheme the CCD divided into exposure regions and storage regions. The shutter is opened, a source spectrum is exposed, the shutter is closed, the CCD is shifted up by 1330 rows to move the sky spectrum into the storage regions while the telescope or chopping mirror redirects the background direction into the exposure regions. The shutter is opened, the background spectrum exposed, shutter closed, and CCD shifted back 1330 rows to bring the source regions into the exposure area and move the background spectra into the storage area. This cycle is repeated until the source and background spectra are sky-noise dominated at read-out. This approach works well only if the time for clocking through the CCD is much shorter than any airglow variation timescales. For this application cycle times depend on the airglow temporal power spectrum. We use measurement of $\mathrm{OH}$ Meinel bands as guidance, since $\mathrm{OI} 5770 \AA$ and $\mathrm{O}_{2}$ bands exhibit behavior similar to $\mathrm{OH}^{8}$. Based on the power spectrum amplitude and slope of ${ }^{9}$, an observing night of 8 hours must be sampled at least 300-1000 times, or roughly one cycle per minute, to reduce the residual subtraction error. The telescope drive, which can slew 10 arcminutes and stabilize with high accuracy in seconds, will be slaved to the camera controller. Guide stars will be established in both 
regions, and the standard guider modified to accommodate chopping. If we are successful, "source" minus "background" spectra residuals will be produced by true sky variations, including that of the IGM emission. While we propose a strategy below to locate emission, it may be detected either in the source or background images, as positive or "negative" emission. A chopping distances of 1-10 arcminutes is required so that typical structures as seen in Figure 2 are clearly separated in velocity space.
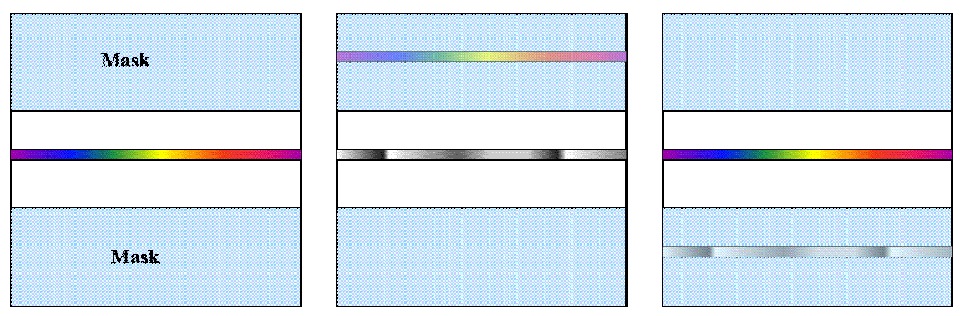

Fig. 8. Schematics showing shift and nod on the CCD.

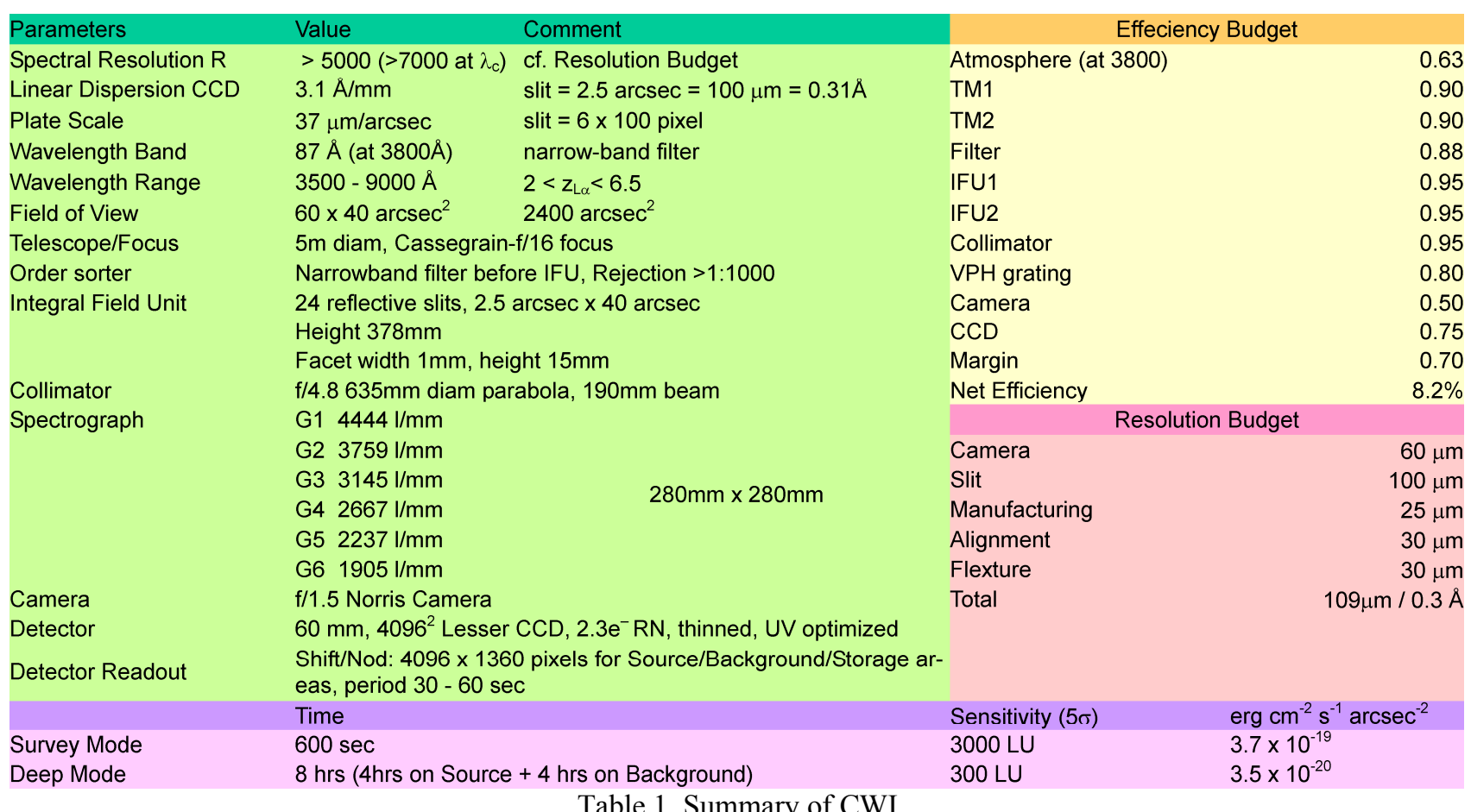

\section{INSTRUMENT MODELING}

We performed a preliminary modeling of the instrument for understanding the systematics and data reduction. A $60 \mathrm{x}$ $60 \operatorname{arcsec}^{2}$ region shown in Figure 9, corresponding to $360 \times 360$ pixel $^{2}$ modeled on Cantalupo simulation was used to model the instrument. 6000s exposure was assumed. For each pixel of the Cantalupo simulation, Ly $\alpha$ photons $(\mathrm{z}=2.086)$ were distributed into wavelength bin according to Figure 10 which was modeled on possible detection of Ly $\alpha$ fluorescence from DLA ${ }^{10}$. Next sky emission spectra sown in Figure 11 were added to each pixel at 10000 bins $(0.01 \AA)$ to produce the source field. Another field with just sky emission spectra and no Lya photons was produced for the background field. Poisson noise was added to both fields and the fields were turned into a long slit and diffracted to produce Figure 12 which is $1000 \times 5184$ pixel $^{2}$ represents only a part of the CCD. The resulting data was reduced to produce $72 \times 72$ pixel $^{2}$ (spatial resolution of 5/6 arcsec, 42micron) image with spectra information in 333 bins $(0.3 \AA)$. 
Figure 13 shows a reconstructed image at $3750 \AA$. Figure 14 shows a spectra of one of the bright pixels shown in lower left corner of Figure 9.

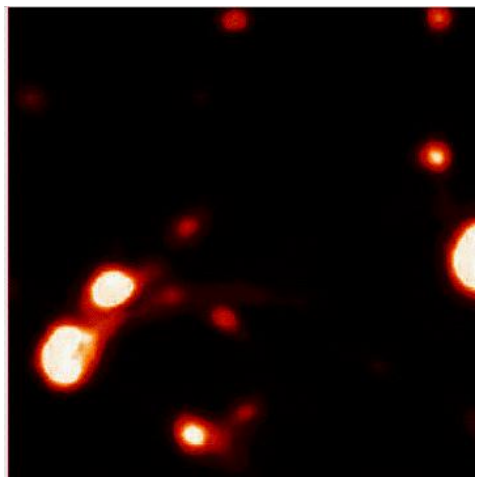

Fig. 9 Input: $60 \times 60 \operatorname{arcsec}^{2}$ region modeled on ${ }^{4}$ simulation.

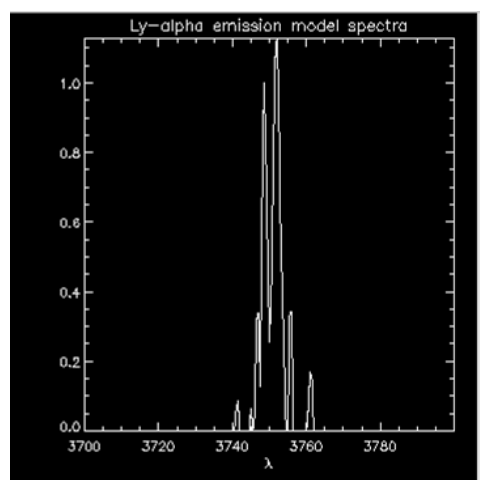

Fig. 10 Input: the wavelegth distribution of Ly $\alpha$ photons $(\mathrm{z}=2.086)$ modeled on observation by ${ }^{10}$.

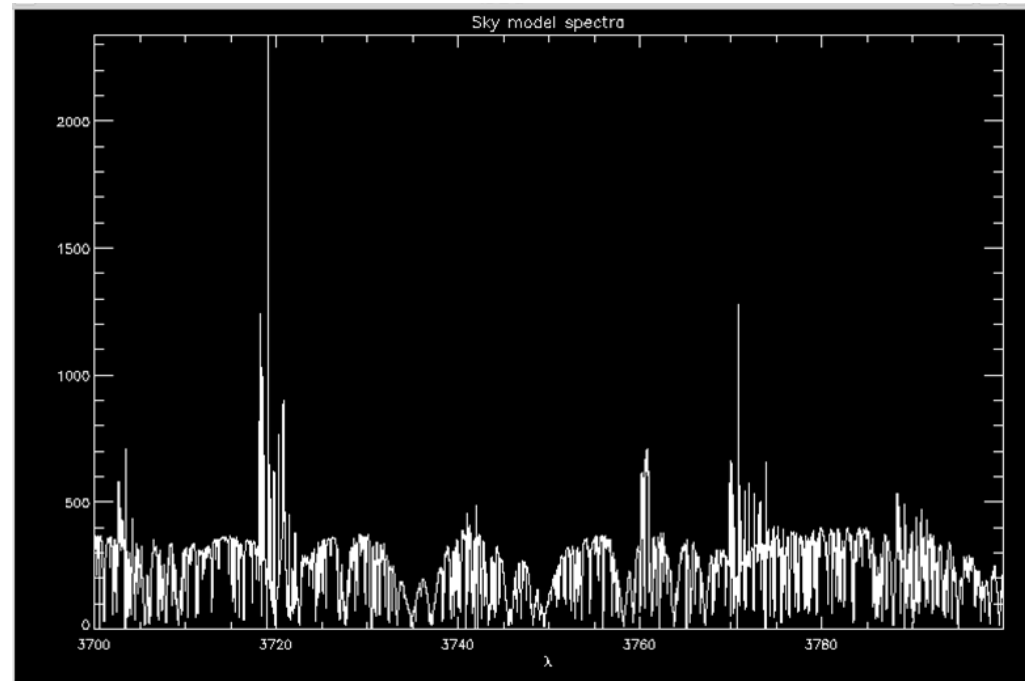

Fig. 11 Input: Simulated sky spectra. 


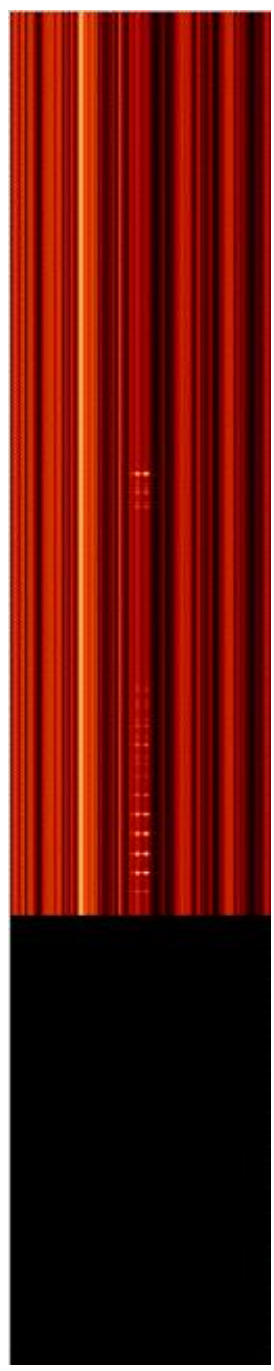

Fig. 12. Output: Part of the CCD is $1000 \times 5184$ pixel $^{2}$. corresponds to wavelength pixel, y-axis corresponds to spatial pixel, TOP $1 / 3$ is sky, MIDDLE $1 / 3$ is target field, BOTTOM $1 / 3$ is charge storage region.

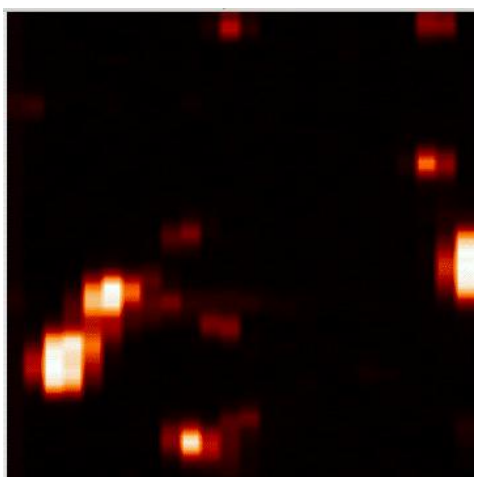

Fig. 13. Output: Output target field with sky subtracted at $3750 \AA$. 


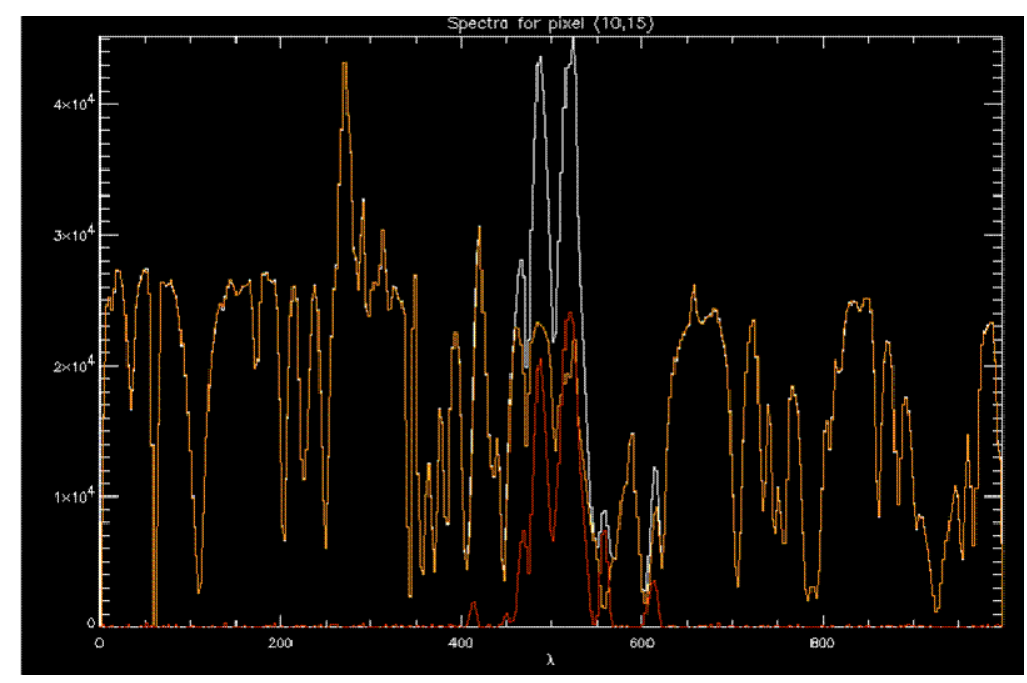

Fig. 14. Output: Spectra for the pixel corresponding to the bright blob in lower left corner of Figure 9, white line is target field spectra, yellow line is sky spectra, red line is the resulting Ly $\alpha$ emission spectra

\section{REFERENCES}

1. $\quad$ K. L. Adelberger, "Galaxies, intergalactic absorption lines, and feedback at high redshift," in IAU Colloq. 199: Probing Galaxies through Quasar Absorption Lines, (2005), pp. 341-348.

2. V. I. Krassovsky, N. N. Shefov, and V. I. Yarin, "Atlas of the airglow spectrum 3000-12400 A,," Planetary and Space Science 9, 883 (1962).

3. R. G. Stockwell, and R. P. Lowe, "Airglow imaging of gravity waves, 1, Results from a small network of $\mathrm{OH}$ nightglow scanning imagers," Journal of Geophysical Research 106, 17185-17204 (2001).

4. S. Cantalupo, C. Porciani, S. J. Lilly, and F. Miniati, "Fluorescent Lyalpha Emission from the High-Redshift Intergalactic Medium," Astrophysical Journal 628, 61-75 (2005).

5. S. C. Barden, J. A. Arns, and W. S. Colburn, "Volume-phase holographic gratings and their potential for astronomical applications," in Proc. SPIE Vol. 3355, p. 866-876, Optical Astronomical Instrumentation, Sandro D'Odorico; Ed., (1998), pp. 866-876.

6. H. W. Epps, "Camera designs for the Keck Observatory LRIS and HIRES spectrometers," in Instrumentation in astronomy VII; Proceedings of the Meeting, Tucson, AZ, Feb. 13-17, 1990 (A91-29601 11-35). Bellingham, WA, Society of Photo-Optical Instrumentation Engineers, 1990, p. 550-561., (1990), pp. 550-561.

7. K. Glazebrook, and J. Bland-Hawthorn, "Microslit Nod-Shuffle Spectroscopy: A Technique for Achieving Very High Densities of Spectra," Publications of the Astronomical Society of the Pacific 113, 197-214 (2001).

8. $\quad$ D. Barbier, "The airglow," Vistas in Astronomy 2, 929-939 (1956).

9. J. H. Hecht, S. K. Ramsay Howat, R. L. Walterscheid, and J. R. Isler, "Observations of spectra intensity fluctuations of the OH Meinel nightglow during ALOHA-93," Geophysical Research Letters 22, 2873-2876 (1995).

10. K. L. Adelberger, C. C. Steidel, J. A. Kollmeier, and N. A. Reddy, "Possible Detection of Lyalpha Fluorescence from a Damped Lyalpha System at Redshift z 2.8," Astrophysical Journal 637, 74-79 (2006). 